\title{
Efeito do clareamento caseiro contendo diferentes espessantes sobre a cor do esmalte dentário bovino
}

\author{
Effect of homemade bleaching containing different thickeners on the color of bovine tooth enamel
}

Efecto del blanqueamiento casero que contiene diferentes espesantes sobre el color del esmalte dental bovino

Recebido: 18/08/2021 | Revisado: 26/08/2021 | Aceito: 31/08/2021 | Publicado: 03/09/2021

Leon Tovar Leite

ORCID: https://orcid.org/0000-0002-1021-3419 Universidade Federal da Paraíba, Brasil

E-mail: leon.leite7@gmail.com

Beatriz Barros Viana

ORCID: https://orcid.org/0000-0001-8105-1069 Universidade Federal da Paraíba, Brasil

E-mail: beatriz.barros@academico.ufpb.br

Lucas Oliveira Macêdo

ORCID: https://orcid.org/0000-0002-4920-4878 Universidade Federal da Paraíba, Brasil E-mail: lucasmacedo002@gmail.com

Renally Bezerra Wanderley e Lima ORCID: https://orcid.org/0000-0003-4477-7850 Faculdade Nova Esperança, Brasil E-mail: renallywanderley@gmail.com

Ana Karina Maciel de Andrade

ORCID: https://orcid.org/0000-0003-4520-5176 Universidade Federal da Paraíba, Brasil E-mail: kamandrade@hotmail.com

Sônia Saeger Meireles

ORCID: https://orcid.org/0000-0001-7328-2991 Universidade Federal da Paraíba, Brasil E-mail: soniasaeger@hotmail.com

Robinsom Viégas Montenegro

ORCID: https://orcid.org/0000-0002-8253-5361 Universidade Federal da Paraíba, Brasil E-mail: rvmontenegro@hotmail.com

Raquel Venâncio Fernandes Dantas ORCID: https://orcid.org/0000-0002-7556-9408 Universidade Federal da Paraíba, Brasil E-mail: raquelvenancio@hotmail.com

Rosângela Marques Duarte ORCID: https://orcid.org/0000-0003-4369-2951 Universidade Federal da Paraíba, Brasil E-mail: rose_marquesd@hotmail.com

\begin{abstract}
Resumo
O objetivo do artigo foi avaliar as alterações de cor do esmalte dentário bovino clareado com peróxido de carbamida a 10\% contendo diferentes espessantes: Carbopol, Natrosol e Carboximetilcelulose. O preparo dos espécimes foi realizado através do uso de um disco diamantado dupla face com o intuito de se obter corpos de prova com $1 \mathrm{~mm}$ de espessura de esmalte. Cada amostra foi submetida ao processo de manchamento com chá preto ao longo de seis dias e posterior imersão em saliva artificial para estabilização da cor. Foi realizada a divisão dos grupos e o protocolo clareador foi iniciado durante 14 dias (4h/dia), utilizando peróxido de carbamida a 10\% (PC 10\%) com Carbopol, e três agentes clareadores manipulados em farmácia: PC 10\% com Carbopol, Natrosol e Carboximetilcelulose. A avaliação da estabilidade de cor foi realizada através de um espectrofotômetro digital (Vita EasyShade) após o manchamento, bem como posteriormente ao protocolo clareador. A análise estatística foi realizada pelos seguintes testes: Kruskal Wallis e Mann Whitney $(\mathrm{p}<0,05)$. Os resultados revelaram diferenças estatisticamente significantes do PC $10 \%$ comercialmente vendido em relação aos parâmetros $\Delta \mathrm{L}, \Delta \mathrm{a}, \Delta \mathrm{b}$ e $\Delta \mathrm{E}$ quando comparado aos produtos manipulados em farmácia. Não houve diferença estatística quando comparados os produtos manipulados entre si. Conclui-se que os clareadores manipulados com diferentes espessantes (Carbopol, Natrosol, Carboximetilcelulose) apresentaram eficácia clareadora inferior ao produto já comercializado.
\end{abstract}

Palavras-chave: Peróxido de carbamida; Clareamento dental; Espessantes; Cor. 


\begin{abstract}
The article aimed to evaluate the color changes of bovine tooth enamel bleached with $10 \%$ carbamide peroxide containing different thickeners: Carbopol, Natrosol, and Carboxymethylcellulose. The preparation of specimens was performed using a double-faced diamond disc to obtain $1 \mathrm{~mm}$-thick enamel specimens. Each sample was subjected to the staining process with black tea over six days and then immersed in artificial saliva to stabilize the color. The groups were divided and the bleaching protocol was started for 14 days (4h/day), using $10 \%$ carbamide peroxide (PC 10\%) with Carbopol, and three bleaching agents manipulated in a pharmacy: PC $10 \%$ with Carbopol, Natrosol, and Carboxymethylcellulose. The evaluation of color stability was performed using a digital spectrophotometer (Vita EasyShade) after staining, as well as after the bleaching protocol. Statistical analysis was performed using the following tests: Kruskal Wallis and Mann Whitney $(\mathrm{p}<0.05)$. The results revealed statistically significant differences for the commercially sold PC $10 \%$ concerning the parameters $\Delta \mathrm{L}, \Delta \mathrm{a}, \Delta \mathrm{b}$, and $\Delta \mathrm{E}$ when compared to products handled in a pharmacy. There was no statistical difference when comparing the products handled with each other. It can be concluded that the bleaching agents manipulated with different thickeners (Carbopol, Natrosol, Carboxymethylcellulose) presented lightening efficacy inferior to the product already commercialized.
\end{abstract}

Keywords: Carbamide peroxide; Tooth bleaching; Thickeners; Color.

\title{
Resumen
}

El objetivo del artículo fue evaluar los cambios de color del esmalte dental bovino blanqueado con peróxido de carbamida al $10 \%$ que contiene diferentes espesantes: Carbopol, Natrosol y Carboximetilcelulosa. La preparación de las probetas se realizó mediante un disco de diamante de doble cara para obtener probetas de esmalte de $1 \mathrm{~mm}$ de espesor. Cada muestra se sometió al proceso de tinción con té negro durante seis días y luego se sumergió en saliva artificial para estabilizar el color. Se dividieron los grupos y se inició el protocolo de blanqueo durante 14 días (4h / día), utilizando peróxido de carbamida al 10\% (PC 10\%) con Carbopol, y tres blanqueadores manipulados en farmacia: PC 10\% con Carbopol, Natrosol y Carboximetilcelulosa. La evaluación de la estabilidad del color se realizó mediante un espectrofotómetro digital (Vita EasyShade) después de la tinción, así como después del protocolo de blanqueo. El análisis estadístico se realizó mediante las siguientes pruebas: Kruskal Wallis y Mann Whitney (p <0.05). Los resultados revelaron diferencias estadísticamente significativas para el PC 10\% vendido comercialmente en relación con los parámetros $\Delta \mathrm{L}, \Delta \mathrm{a}, \Delta \mathrm{b}$ y $\Delta \mathrm{E}$ en comparación con los productos manipulados en una farmacia. No hubo diferencia estadística al comparar los productos manipulados entre sí. Se puede concluir que los blanqueadores manipulados con diferentes espesantes (Carbopol, Natrosol, Carboximetilcelulosa) presentaron una eficacia aclarante inferior al producto ya comercializado.

Palabras clave: Peróxido de carbamida; Blanqueamiento de dientes; Espesantes; Color.

\section{Introdução}

Atualmente, a aparência e a cor dos dentes têm se tornado uma preocupação bastante comum na população adulta jovem, movida pela busca por tratamentos que melhorem a estética e consequentemente a autoestima (Joiner \& Luo, 2017; Pavicic et al., 2020), visto que dentes agradáveis desempenham um papel essencial e interferem diretamente nas interações sociais (Bersezio et al., 2018; Penha et al., 2018).

A busca por um sorriso estético tem estimulado a procura por tratamentos eficazes e alternativas para aumentar sua atratividade (Borges et al., 2014), entretanto o clareamento dental é o tratamento não restaurador mais comumente prescrito para o manejo de dentes escurecidos (Morgan et al., 2015). Suas vantagens se dão por apresentar um protocolo caracterizado pela ausência de remoção de tecido dentário, assim como o baixo tempo clínico demandado (AlOtaibi et al., 2020; Kothari et al., 2020). Vale salientar que, tratamentos minimamente invasivos poupam pacientes de uma ampla gama de complicações técnicas e biológicas que requerem manutenção de longo prazo e/ou intervenções subsequentes (Kothari et al., 2020).

O processo de escurecimento dentário é justificado através da teoria do cromóforo, onde as ligações duplas conjugadas fornecem cor às moléculas. Caso ocorra uma quebra dessas ligações, as moléculas se tornam transparentes (Marquillas et al., 2020). No clareamento, os radicais livres produzidos pela decomposição do peróxido de hidrogênio, principal componente dos géis clareadores, conseguem penetrar na estrutura dentária e oxidar essas moléculas, promovendo o efeito clareador (Borges et al., 2014; Penha et al., 2018; Zanolla et al., 2017). 
Os géis clareadores contêm em suas formulações polímeros espessantes com alto peso molecular utilizados como um agente de viscosidade, fornecendo ao produto uma consistência de gel e permitindo um contato mais próximo do agente clareador com a superfície do dente, prolongando a liberação de radicais produzidos pelo peróxido de hidrogênio (Gouveia et al., 2016, 2020). O espessante mais utilizado junto ao gel de peróxido de carbamida é o Carbopol, um polímero de carboxipolimetileno, iônico e ácido na natureza, sendo um derivado do ácido carboxílico (Gouveia et al., 2016; Silva et al., 2018).

Contudo, a ação sinérgica de espessantes e radicais livres liberados pela decomposição do peróxido pode potencializar a degradação orgânica da matriz polimérica e, consequentemente, prejudicar o brilho da superfície, principalmente em associação com o espessante iônico (Gouveia et al., 2016). Estudos recentes também têm atribuído à sensibilidade dentária pós tratamentos clareadores, o baixo $\mathrm{pH}$ e alta viscosidade do Carbopol, fatores que provocam alterações na estrutura, composição e morfologia do esmalte, influenciando assim na sintomatologia dolorosa do paciente (Gouveia et al., 2020; Públio et al., 2019; Silva et al., 2018). Uma das alterações na morfologia da estrutura dentária é a diminuição nos valores de microdureza de superfície do esmalte (Gouveia et al., 2020), um dos fatores de incentivo à busca por espessantes alternativos.

Outro espessante disponível é o Natrosol (hidroxietilcelulose), agente derivado da celulose e amplamente utilizado em vários materiais para engrossar, estabilizar e emulsificar (Públio et al., 2019). Por apresentar uma excelente compatibilidade com diferentes tipos de compostos, seu uso em agentes clareadores também foi proposto, principalmente por apresentar características não iônicas e seu uso ser recomendado em substâncias ácidas por possuir uma estabilidade à variação do pH $(2,0-12,0)($ Gouveia et al., 2016).

Outros derivados da celulose são amplamente aplicados na obtenção de produtos farmacêuticos, cosméticos e alimentícios (Casaburi et al., 2017; Javanbakht \& Shaabani, 2019). A carboximetilcelulose (CMC), éter derivado da celulose e comercializado na forma sólida como sal de carboximetilcelulose de sódio, é um destes compostos. A CMC, por ser de fácil dissolução e apresentar grande capacidade espessante e estabilizante (Landim et al., 2015), além de possuir propriedades hidrofílicas, bioadesivas, apresentar sensibilidade a variações de pH, ser atóxico e ter capacidade de formação de gel (Javanbakht \& Shaabani, 2019), vem sendo utilizada também na composição de agentes clareadores.

Apesar do amplo uso dos agentes espessantes anteriormente mencionados, suas influências nas propriedades de superfície do esmalte não são profundamente investigadas na literatura. Um estudo prévio mostrou que o carbopol utilizado sem associação do clareador diminuiu a microdureza do esmalte dental (Silva, 2018). Outro ponto a ser mencionado diz respeito ao fato de que cada fabricante desenvolve sua própria formulação em diferentes níveis de concentrações e níveis de viscosidade, podendo ocasionar resultados destoantes nas estruturas dentárias.

Desta forma, o objetivo deste estudo in vitro foi avaliar o efeito causado com relação à alteração de cor do esmalte dentário bovino clareado com peróxido de carbamida a $10 \%$ contendo diferentes espessantes: Carbopol, Natrosol e Carboximetilcelulose manipulados em farmácia e comparados com peróxido de carbamida a 10\% com carbopol industrial. A hipótese nula foi de que não havia diferença na cor do esmalte dentário bovino após uso de produtos para clareamento contendo diferentes espessantes.

\section{Metodologia}

Estudo experimental in vitro, realizado pelo método indutivo, com técnica de documentação direta com pesquisa em laboratório (Marconi \& Lakatos, 2019).

\section{Preparo dos espécimes}

Inicialmente, incisivos bovinos foram extraídos e armazenados em solução aquosa contendo timol $0,1 \%$ até o preparo dos mesmos. O debridamento manual dos elementos dentários foi realizado com o auxílio de lâminas de bisturi e, após a limpeza, os 
mesmos foram armazenados em água destilada. Posteriormente, foi realizada a separação da porção coronária com auxílio de um disco diamantado dupla face (KG Sorensen, Ind. Com. Ltda, Barueri, SP, Brasil) acoplado em micromotor de baixa rotação (Dabi Atlante; Ribeirão Preto, SP, Brasil) e em constante irrigação com água. Após a coleta, as coroas dos elementos foram armazenadas em água destilada.

Para a confecção dos espécimes, cortes foram realizados na porção coronária em duplo sentido, inciso-cervical e mésiodistal com o uso de disco diamantado (4" $\times 012 \times 1 / 2$, Buehler, Illinois, USA), proporcionando uma padronização dos fragmentos com uma área superficial de $16 \mathrm{~mm}^{2}$ (4x4 mm) e $3 \mathrm{~mm}$ de altura, sendo $1 \mathrm{~mm}$ de espessura de esmalte (Silva et al., 2018).

\section{Manchamento dentário}

Cada amostra foi imersa em 2,5 mL de solução de chá preto (Leão Junior S.A., Curitiba, PR, Brasil) (1,6g de erva + 100 $\mathrm{mL}$ de água destilada fervente) ao longo de seis dias, com trocas realizadas a cada 24 horas (Gouveia et al., 2020). Após o período de imersão, as amostras foram armazenadas em saliva artificial (CaCl2, $\mathrm{KH} 2 \mathrm{PO} 4$, Mucina, $\mathrm{KCl}, \mathrm{NaCl}$, Tris Buffer e $\mathrm{H} 2 \mathrm{O}$ ) (Dilecta, João Pessoa, PB, Brasil) durante duas semanas para estabilização da cor. A borra de chá preto formada sobre o bloco dentário foi removida através do uso de uma taça de borracha com pedra pomes e água (proporção 2:1) em baixa rotação por 30 segundos. Após isso, foi realizada a leitura da cor das amostras conforme os parâmetros descritos no método de análise.

\section{Protocolo de aplicação do tratamento clareador/espessante}

As amostras foram divididas em 4 grupos ( $\mathrm{n}=10)$, recebendo tratamentos clareadores de acordo com os grupos a quais pertenciam: Peróxido de carbamida 10\% (PC 10\%) com Carbopol (FGM, Whiteness Perfect, Joinville, SC, Brasil), PC 10\% com Carbopol (Alquimia, João Pessoa, PB, Brasil), PC 10\% com Natrosol (Alquimia, João Pessoa, PB, Brasil) e PC 10\% com Carboximetilcelulose (Alquimia, João Pessoa, PB, Brasil) (Tabela 1).

Ao longo de 14 dias, os espécimes receberam uma sessão de tratamento diário durante 4 horas. O gel clareador $(0,02 \mathrm{~mL})$ foi aplicado na superfície das amostras com uma gota de saliva artificial com auxílio de pipetas graduadas (Digipet, Curitiba, Brasil) de maneira a preencher toda a superfície dos corpos de prova.

Transcorrido o tempo proposto de 4 horas, os espécimes foram lavados abundantemente em água corrente, secos com papel absorvente (Kleenex - Kimberly-Clark, Brasil) e armazenados em 3,5 ml de solução de saliva artificial $(\mathrm{pH}=7,0)$ em estufa a $37^{\circ} \mathrm{C}\left( \pm 2^{\circ} \mathrm{C}\right)$ até a próxima sessão clareadora (Gouveia et al., 2016).

Tabela 1 - Divisão dos grupos e o produto utilizado.

\begin{tabular}{c|l}
\hline GRUPOS & \multicolumn{1}{c}{ PRODUTO UTILIZADO } \\
\hline PC 10\% & Clareamento com peróxido de carbamida 10\% - FGM \\
\hline PC 10\% /C & Clareamento com peróxido de carbamida 10\% manipulado com Carbopol - ALQUIMIA \\
\hline PC 10\% /N & Clareamento com peróxido de carbamida 10\% manipulado com Natrosol- ALQUIMIA \\
\hline PC 10\% /CMC & Clareamento com peróxido de carbamida 10\% manipulado com Carboximetilcelulose - ALQUIMIA \\
\hline
\end{tabular}

Fonte: Autores.

\section{Método de análise do experimento}

As análises de cor foram realizadas após o manchamento e posteriormente aos tratamentos clareadores realizados, utilizando um espectrofotômetro digital (Vita Easyshade, Vita ZahnFabrick, Bad Säckingen, Alemanha) pelo sistema CIELab. 
Previamente, o equipamento foi calibrado de acordo com as indicações do fabricante, onde cada amostra foi colocada sobre uma folha de papel A4 branca, em uma superfície lisa e plana, em local iluminado e climatizado, com a ponta do espectrofotômetro posicionada no centro da amostra e a marcação localizada à direita do operador. Para a padronização, todas as leituras foram realizadas no turno da manhã em temperatura controlada de $25^{\circ} \mathrm{C}$. Em cada análise, foram registradas três leituras das amostras, obtendo valores das coordenadas $\mathrm{L}^{*}, \mathrm{a}^{*}$ e $\mathrm{b}^{*}$, sendo expressas por $\Delta \mathrm{L}^{*}, \Delta \mathrm{a}^{*}$ e $\Delta \mathrm{b}^{*}$, parâmetros que representam a subtração da leitura final e inicial. A diferença de cores $(\Delta \mathrm{E})$ foi calculada entre a primeira e a segunda leitura de acordo com a equação: $\Delta \mathrm{Eab}=\left[\left(\Delta \mathrm{L}^{*}\right)^{2}+\left(\Delta \mathrm{a}^{*}\right)^{2}+\left(\Delta \mathrm{b}^{*}\right)^{2}\right]^{1 / 2}$.

\section{Análise estatística}

Os resultados deste estudo foram tabulados e a análise estatística realizada por meio do software SPSS versão 24.0 (SPSS, Chicago, USA). Os valores das propriedades ópticas foram submetidos ao teste estatístico de Kruskal Wallis. Para comparações entre dois produtos, foi realizado o teste de Mann Whitney, estabelecendo-se $\mathrm{p}<0,05$.

\section{Resultados}

$\mathrm{Na}$ Tabela 2 são apresentados os valores da variação de cor $\Delta \mathrm{L}, \Delta \mathrm{a}, \Delta \mathrm{b}$ e $\Delta \mathrm{E}$. Foi possível observar diferenças estatisticamente significantes $(\mathrm{p}<0,05)$ entre os grupos nas variáveis $\Delta \mathrm{L}, \Delta \mathrm{a}, \Delta \mathrm{b}, \Delta \mathrm{E}$. Além disso, quando analisada a variação de cor $\Delta \mathrm{E}$, o grupo representado pelo clareador da FGM (PC 10\%) apresentou maiores alterações de cor em relação aos demais grupos, indicando um maior clareamento. Esse padrão se repetiu para os parâmetros $\Delta \mathrm{L}, \Delta \mathrm{a}$ e $\Delta \mathrm{b}$. Os grupos $\mathrm{PC} 10 \% / \mathrm{C}, \mathrm{PC}$ $10 \%$ /N e PC 10\% /CMC não apresentaram diferenças estatisticamente significantes entre si (p>0,05).

Tabela 2 - Média e desvio-padrão da variação de cor através de $\Delta \mathrm{L}, \Delta \mathrm{a}, \Delta \mathrm{b}$ e $\Delta \mathrm{E}$ de acordo com o tipo de espessante utilizado. João Pessoa, PB, 2020.

\begin{tabular}{c|c|c|c|c}
\hline GRUPOS & $\boldsymbol{\Delta L}$ & $\boldsymbol{\Delta a}$ & $\boldsymbol{\Delta b}$ & $\mathbf{\Delta}$ \\
\hline $\mathbf{P C ~ 1 0 \%}$ & $32,42(4,93) \mathrm{a}$ & $-15,35(2,28) \mathrm{a}$ & $-17,65(5,21) \mathrm{a}$ & $40,37(4,66) \mathrm{a}$ \\
\hline PC 10\% /C & $8,71(5,32) \mathrm{b}$ & $-5,48(1,83) \mathrm{b}$ & $-7,38(2,70) \mathrm{b}$ & $13,57(3,52) \mathrm{b}$ \\
\hline PC 10\%/N & $10,10(11,62) \mathrm{b}$ & $-6,15(4,34) \mathrm{b}$ & $-3,20(3,08) \mathrm{b}$ & $15,30(8,37) \mathrm{b}$ \\
\hline PC 10\% /CMC & $15,34(13,52) \mathrm{b}$ & $-6,93(5,80) \mathrm{b}$ & $-3,91(4,74) \mathrm{b}$ & $19,78(11,66) \mathrm{b}$ \\
\hline $\mathbf{P}$ & $<0,001$ & $<0,001$ & $<0,001$ & $<0,001$ \\
\hline
\end{tabular}

Letras distintas na vertical diferem entre si $(\mathrm{p}<0,05)$. Fonte: Autores.

\section{Discussão}

Alterações de cor representam o desequilíbrio estético mais comum do sorriso, visto que dentes brancos são considerados um sinal de cuidado e beleza, tendo grande impacto na autoestima dos pacientes (Penha et al., 2018). O clareamento é um procedimento que ilumina a cor do elemento dentário através da aplicação de um agente químico, oxidando a pigmentação orgânica do dente (Mounika et al., 2018).

O Peróxido de carbamida a $10 \%$ tem sido o agente clareador mais comumente utilizado em tratamentos de clareamento caseiros (Qusti et al., 2020). Ele decompõe-se em peróxido de hidrogênio e ureia quando aplicado nas superfícies dentais, sendo a concentração de peróxido de hidrogênio aproximadamente um terço da porcentagem do peróxido de carbamida. É considerado o agente ativo do clareador devido sua redução em água e oxigênio. Já a ureia, como se transforma em amônia e dióxido de carbono, eleva o pH facilitando a dissociação do agente clareador (Luque-Martinez et al., 2016).

O presente estudo avaliou a influência de agentes espessantes utilizados no peróxido de carbamida a $10 \%$ nas variações de cor do esmalte bovino através do sistema CIELab. Na escala de cor CIELab, o eixo L* varia de 0 a 100 e representa o grau 
de luminosidade da cor, considerando 0 totalmente preto e 100 totalmente branco. O parâmetro a* representa a variação entre o verde (a-) e o vermelho (a+), e b* representa a variação entre o azul (b-) e o amarelo (b+) (Rodrigues et al., 2020). Ou seja, o aumento da luminosidade (aumentando $\mathrm{L}^{*}$ ) e a redução do tom amarelado (diminuindo b*) são os principais indutores do clareamento dental (Pustina-Krasniqi et al., 2017).

Os resultados do presente estudo revelaram que os produtos manipulados em farmácia, sejam constituídos por agentes espessantes representados pelo Carbopol, Natrosol ou Carboximetilcelulose, apresentaram resultados divergentes dos obtidos do PC 10\%, apresentando valores inferiores e evidenciando uma menor ação clareadora. Os valores elevados de $\Delta \mathrm{E}$ do grupo PC $10 \%$ indicam um considerável clareamento, assim como o aumento da luminosidade $(\Delta \mathrm{L})$ e a diminuição do eixo b. Quando analisados PC 10\% e PC 10\% /C, essa diferença pode ser justificada por uma possível alteração na eficácia do agente clareador manipulado em farmácia. A composição do peróxido industrial é: Peróxido de Carbamida, carbopol neutralizado, nitrato de potássio, fluoreto de sódio, umectante (Glicol), água deionizada. Já os detalhes dos produtos manipulados não foram fornecidos. A instabilidade do peróxido foi relatada na literatura e é possível de ocorrer. Seja qual for o tipo, a concentração e a forma de apresentação do clareador, todos são sensíveis às condições de manipulação e armazenamento, podendo perder significativamente seu poder de ação com o tempo e quando expostos à luz, ao calor e ao meio ambiente (Soares et al., 2006). Freitas (2015) concluiu que das sete formulações analisadas, duas estavam com o teor de peróxido de carbamida abaixo do valor especificado de $78 \%$. Complementa ainda a necessidade de um maior controle por partes das farmácias de manipulação de forma a dispensar clareadores dentais eficazes, seguros e com qualidade.

Os produtos oriundos de farmácia de manipulação (PC 10\% /C e PC 10\% /N) não apresentaram diferenças entre si, mas obtiveram resultados inferiores quando comparados a PC 10\% industrial, apresentando assim, diferenças estatisticamente significantes. O carbopol utilizado no gel clareador industrializado nem sempre é o mesmo do manipulado. Além disso, dois fatores representam condição crítica para a efetividade do produto clareador e também influenciam na sensibilidade pósoperatória: o pH do gel clareador que pode provocar até mesmo desmineralização das estruturas dentais e o nível de neutralização do carbopol, que é um polímero de caráter ácido (Soares et al., 2006). De fato, existem inúmeras fórmulas de clareamento comercialmente disponíveis, com diferentes composições e concentrações, feitas por diferentes fabricantes. No estudo de Martin et al. (2007) nem os agentes clareadores preparados em farmácias de manipulação, nem o produto industrializado apresentou a concentração esperada de peróxido, entretanto embora o produto comercial avaliado não tenha atingido a maior concentração média quando em comparação com os produtos manipulados, foi o mais adequado quando se considerou os valores máximo e mínimo, porque sua concentração foi a mais constante e com o menor desvio padrão.

Os resultados apresentados por Públio et al. (2019), onde o clareamento com peróxido de carbamida a 10\% contendo o espessante Carbopol (FGM) e Natrosol (manipulado em farmácia) foram realizados, divergem parcialmente do aqui encontrados. $\mathrm{O}$ referido estudo apresentou ausência de diferenças estatísticas significativas em relação aos parâmetros $\Delta \mathrm{L}$ e $\Delta \mathrm{E}$ para os agentes clareadores/espessantes após 14 dias, mas não para o parâmetro $\Delta b$. Isto pode ter ocorrido devido a divergência de onde foram obtidos os produtos, bem como na metodologia adotada, onde houve a inexistência do manchamento dental prévio ao protocolo clareador. Ademais, o estudo laboratorial presente não obteve a dinâmica no meio oral, diferente de um ensaio clínico que fornece um ambiente adequado para a remineralização dentária (Vieira-Junior et al., 2018) e consequente alteração das propriedades ópticas do elemento dentário, como o aumento acentuado de reflexos (Kwon \& Wertz, 2015), alterações na translucidez, opalescência, fluorescência e opacidade do esmalte e dentina, alterando assim, a cor do elemento dentário (Montenegro et al., 2016).

Além disso, estudos evidenciam que as alterações decorrentes do uso de agentes clareadores podem não estar relacionadas apenas a aplicação do peróxido, mas também para a presença do agente espessante (Gouveia et al.,2016; Silva, 2018). O carbopol 
é de natureza iônica, o que dificulta a incorporação de cristais de hidroxiapatita ao elemento dental, causando alterações de microdureza na superfície devido ao seu baixo pH e sua alta viscosidade, ocasionando a desmineralização (Silva, 2018). A desmineralização induz mudanças morfológicas no esmalte (Gouveia et al., 2020; Tsai et al., 2019) pela remoção dos minerais e aumento da porosidade (Ruiz et al., 2020). Assim, deve ser corretamente tamponado para ser utilizado nos clareadores.

Em seu estudo, Silva et al. (2018) observaram que peróxido de carbamida a 10\% + Natrosol (manipulado) apresentou maior variação de $\Delta \mathrm{E}, \Delta \mathrm{L}$ e $\Delta \mathrm{b}$ quando comparado ao peróxido de carbamida associado ao Carbopol (FGM), divergindo dos resultados obtidos no presente estudo. Isto pode estar fundamentado na distinção da metodologia in situ, seguindo a mesma justificativa supracitada da ação salivar na dinâmica do meio oral. A mudança de cor do peróxido de carbamida contendo Natrosol pode estar associada com um maior tempo de ação do espessante para manter radicais livres de peróxido de hidrogênio na estrutura dentária, e/ou devido ao $\mathrm{pH}$ e as espécies oxidativas reativas dos produtos (Públio et al., 2019).

Resultados diferentes foram descritos no trabalho de Gouveia et al. (2020) em que não houve diferenças estatísticas nos parâmetros de $\Delta \mathrm{E}, \Delta \mathrm{L}$ e $\Delta \mathrm{b}$ quando comparado o peróxido de carbamida a $10 \%$ associado ao Carbopol comercializado e o manipulado, podendo ser justificado em razão da manipulação do produto que pode conter alguma concentração e/ou formulação diferente. Esses detalhes muitas vezes não são acessíveis ao pesquisador, levando a acreditar que várias alterações podem ter ocorrido, inclusive da matéria prima ser diferente e até mesmo o armazenamento.

Há uma escassez de estudos na literatura sobre o uso dos espessantes alternativos e suas possíveis alterações na cor da superfície do esmalte dentário, principalmente no que diz respeito ao uso da Carboximetilcelulose. $\mathrm{O}$ fato de o carbopol ter sido relatado na literatura como capaz de alterar propriedades de superfície do elemento dentário, impulsionou diferentes estudos com espessantes alternativos. Mais estudos devem ser realizados para elucidar a ação desses compostos, usados até de maneira isolada, para saber sua real influência nas alterações de cor dentária e em outras propriedades dos tecidos dentários.

\section{Considerações Finais}

De acordo com os resultados obtidos neste estudo in vitro, foi possível concluir que os clareadores manipulados com diferentes espessantes (Carbopol, Natrosol, Carboximetilcelulose) tiveram comportamento equivalente entre si e eficácia clareadora inferior ao produto já comercializado.

\section{Referências}

AlOtaibi, G., AlMutairi, M., AlShammari, M., AlJafar, M., \& AlMaraikhi, T. (2020). Prevalence and public knowledge regarding tooth bleaching in Saudi Arabia. Journal of Family Medicine and Primary Care, 9(7), 3729-3732.

Bersezio, C., Martín, J., Herrera, A., Loguercio, A., \& Fernández, E. (2018). The effects of at-home whitening on patients’ oral health, psychology, and aesthetic perception. BMC Oral Health, 18(1), 1-10.

Borges, A., Zanatta, R., Barros, A., Silva, L., Pucci, C., \& Torres, C. (2014). Effect of Hydrogen Peroxide Concentration on Enamel Color and Microhardness. Operative Dentistry, 40(1), 96-101.

Casaburi, A., Montoya Rojo, Ú., Cerrutti, P., Vázquez, A., \& Foresti, M. L. (2017). Carboxymethyl cellulose with tailored degree of substitution obtained from bacterial cellulose. Food Hydrocolloids, 75, 147-156.

Freitas, C. M. D. (2015). Teor de peróxido de carbamida em clareadores dentais manipulados na cidade de Cascável-PR. [Monografia, Faculdade Assis Gurgacz].

Gouveia, T. H. N., de Souza, D. F. S., Aguiar, F. H. B., Ambrosano, G. M. B., \& Lima, D. A. N. L. (2020). Effect of ammonium acryloyldimethyltaurate copolymer on the physical and chemical properties of bleached dental enamel. Clinical Oral Investigations, 24(8), 2701-2711.

Gouveia, T. H. N., Públio, J. do C., Ambrosano, G. M. B., Paulillo, L. A. M. S., Aguiar, F. H. B., \& Lima, D. A. N. L. (2016). Effect of at-home bleaching with different thickeners and aging on physical properties of a nanocomposite. European Journal of Dentistry, 10(01), $082-091$.

Javanbakht, S., \& Shaabani, A. (2019). Carboxymethyl cellulose-based oral delivery systems. International Journal of Biological Macromolecules, 133, 21-29. $\mathrm{h}$ 
Joiner, A., \& Luo, W. (2017). Tooth colour and whiteness: A review. Journal of Dentistry, 67, S3-S10.

Krasniqi, T. P., Shala, K., Staka, G., Bicaj, T., Ahmedi, E., \& Dula, L. (2017). Lightness, chroma, and hue distributions in natural teeth measured by a spectrophotometer. Journal of Medical Sciences, 11(1), 36-40.

Kothari, S., Jum'ah, A. A., Gray, A. R., M. Lyons, K., Yap, M., \& Brunton, P. A. (2020). A randomized clinical trial investigating three vital tooth bleaching protocols and associated efficacy, effectiveness and participants' satisfaction. Journal of Dentistry, 95, 103322.

Kwon, S. R., \& Wertz, P. W. (2015). Review of the mechanism of tooth whitening. Journal of Esthetic and Restorative Dentistry, $27(5), 240-257$.

Landim, L. B., Sampaio, V. S., Souza Junior, E. C., Bonomo, R. C. F., \& Leite, C. X. S. (2015). Avaliação de Diferentes Espessantes nas Propriedades FísicoQuímicas, Sensoriais e Reológicas de Bebida Láctea. Revista Brasileira de Produtos Agroindustriais, 17(1), 87-96.

Luque-Martinez, I., Reis, A., Schroeder, M., Muñoz, M. A., Loguercio, A. D., Masterson, D., \& Maia, L. C. (2016). Comparison of efficacy of tray-delivered carbamide and hydrogen peroxide for at-home bleaching: a systematic review and meta-analysis. Clinical Oral Investigations, 20(7), 1419-1433.

Marconi, M. A., \& Lakatos, E. M. (2019). Fundamentos de Metodologia Científica. Editora Atlas.

Marquillas, C. B., Procaccini, R., Malmagro, M. V., \& Sánchez-Martín, M.-J. (2020). Breaking the rules: tooth whitening by means of a reducing agent. Clinical Oral Investigations, 24(8), 2773-2779.

Martin, J. M. H., Torno, V., Vaz, M. V. S., Vieira, S., Rosa, E. A. R., \& Mazur, R. F. (2007). Specific concentration evaluation of 16\% carbamide peroxide compounded at dispensing pharmacies. Brazilian Oral Research, 21(4), 318-322.

Montenegro, A. K. R. A., Silva, B. M. A. H., Firoozmand, L. M., Lago, A. D. N., \& Costa, J. F. (2016). Alterações das propriedades ópticas do esmalte e da dentina após o clareamento dental - uma revisão de literatura. FOL, 26(2), 75-82.

Morgan, S., Jum'ah, A. A., \& Brunton, P. (2015). Assessment of efficacy and post-bleaching sensitivity of home bleaching using 10\% carbamide peroxide in extended and non-extended bleaching trays. British Dental Journal, 218(10), 579-582.

Mounika, A., Mandava, J., Roopesh, B., \& Karri, G. (2018). Clinical evaluation of color change and tooth sensitivity with in-office and home bleaching treatments. Indian Journal of Dental Research, 29(4), 423.

Pavicic, D. K., Kolceg, M., Lajnert, V., Pavlic, A., \& Spalj, S. (2020). Changes in quality of life induced by tooth whitening are not influenced by global selfesteem: a randomized double-blind placebo-controlled trial. Odontology, 108(1), 143-151.

Penha, K., Sousa, A., Oliveira, C., de Andrade, R., \& Vasconcelos, D. (2018). A swift, easy and cheap protocol to evaluate the tooth bleaching in vitro. Journal of Clinical and Experimental Dentistry, 10(6), e579-84.

Públio, J. do C., Zeczkowski, M., Burga-Sánchez, J., Ambrosano, G. M. B., Groppo, F. C., Aguiar, F. H. B., \& Lima, D. A. N. L. (2019). Influence of different thickeners in at-home tooth bleaching: a randomized clinical trial study. Clinical Oral Investigations, 23(5), 2187-2198.

Qusti, N. Y., Brooks, S. J., \& Brunton, P. A. (2020). In situ study: Effect of a 10\% Carbamide Peroxide whitening gel on enamel mineral density using NanoCT. Medical Science, 24(106), 4731-4745.

Rodrigues, A. P. M., Klein, M. C., Dall Agnol, M. A., \& Rodrigues-Junior, S. A. (2020). In vitro tooth whitening effectiveness of whitening mouth rinses. Brazilian Journal of Oral Sciences, 19(49), e206779.

Ruiz, S., Díaz-Soriano, A., Gallo, W., Perez-Vargas, F., Munive-Degregori, A., \& Mayta-Tovalino, F. (2020). Assessment of Structural Changes in Translucency and Opacity of Tooth Enamel against a Direct Demineralization Process: An In Vitro Study. Journal of International Society of Preventive \& Community Dentistry, 10(4), 473.

Silva, B. G., Gouveia, T. H. N., da Silva, M. de A. P., Ambrosano, G. M. B., Aguiar, F. H. B., \& Lima, D. A. N. L. (2018). Evaluation of home bleaching gel modified by different thickeners on the physical properties of enamel: An in situ study. European Journal of Dentistry, 12(04), 523-527.

Silva, R. C. P. (2018). Efeito do clareamento caseiro contendo diferentes espessantes na mudança de cor e microdureza do esmalte dental. [Monografia, Universidade Estadual de Campinas]. Biblioteca Digital da UNICAMP. http://www.bibliotecadigital.unicamp.br/document/?code=001072343\&opt=4

Soares, C. J., Silva, N. T., Quagliatto, P. S., \& Campos, R. E. (2006). Avaliação clínica de clareamento caseiro com gel de peróxido de carbamida industrializado e manipulado em farmácia. Revista de Odontologia da UNESP, 35(1), 69-74.

Tsai, M.-T., Wang, Y.-L., Yeh, T.-W., Lee, H.-C., Chen, W.-J., Ke, J.-L., \& Lee, Y.-J. (2019). Early detection of enamel demineralization by optical coherence tomography. Scientific Reports, 9(17154), 1-9.

Vieira-Junior, W., Ferraz, L., Pini, N., Ambrosano, G., Aguiar, F., Tabchoury, C., \& Lima, D. (2018). Effect of Toothpaste Use Against Mineral Loss Promoted by Dental Bleaching. Operative Dentistry, 43(2), 190-200.

Zanolla, J., Marques, A., da Costa, D., de Souza, A., \& Coutinho, M. (2017). Influence of tooth bleaching on dental enamel microhardness: a systematic review and meta-analysis. Australian Dental Journal, 62(3), 276-282. 